\title{
De la gonorrhée à la blennorragie : Les grandes étapes historiques
}

\author{
Georges ANDROUTSOS et Lazaros VLADIMIROS
}

Histoire de la Médecine, Faculté de Médecine, Université d’loannina, Grèce

\section{RESUME}

Dans cet article est examiné l'itinéraire historique de la blennorragie et ses modes de traitement. Très probablement il s'agit d'une très ancienne maladie qui pendant des siècles fut confondue avec la syphilis.

Mots clés : gonorrhée, blennorragie, syphilis, traitement, historique

\section{GENERALITES}

Le mot blennorragie provient des mots grecs blennos = visqueux, et le radical rheo = écoulement. II s'agit d'une infection de la muqueuse des organes génitaux des deux sexes avec formation de pus, due à une bactérie pyogène, le gonocoque. Cette maladie se transmet, en règle générale, par le coït et, dans sa phase aiguë, consiste en une urétrite (chez l'homme) ou en une vulvo-vaginite (chez la femme). Très souvent, l'infection se propage dans l'appareil génito-urinaire, s'étend au voisinage et devient chronique. Elle peut déterminer des manifestations pathologiques articulaires, cutanées et oculaires [59]. L'extension de l'infection peut entraîner, rarement, une septicémie.

La blennorragie peut provoquer des escarres des canaux déférents et des trompes de Fallope; chez l'homme elle provoque un rétrécissement du canal urétral donnant des mictions douloureuses à l'origine de l'appellation populaire chaude-pisse et elle peut entraîner dans les deux sexes la stérilité par obstruction des voies de transport des spermatozoïdes et des ovules [54].

\section{UNE MALADIE DE TOUS LES TEMPS ?}

L'origine du germe de la blennorragie est inconnue. Sans doute, il est né par mutation d'une Neisseriacée saprophyte, mais on ne peut pas, en l'état actuel de nos connaissances, dater cet événement. La blennorragie a-t-elle sévi en Grèce dans les temps très anciens ? L'ostéo-archéologie ne peut pas répondre à cette question. Bien que le gonocoque puisse marquer les os par son activité pathogène, ces lésions ne sont pas spécifiques [28].

L'examen critique des anciens témoignages littéraires ne permet pas de trancher : d'une part, le diagnostic de blennorragie est compatible avec certaines descriptions antiques ; d'autre part, aucune de ces descriptions ne l'impose comme seule interprétation possible.

La gonorrhée ou pisse-chaude de François Rabelais (1494-1553), pour la plupart des historiens de la médecine, existait déjà dans l'Antiquité et elle est citée dans les textes les plus anciens de toutes civilisations. Les documents écrits les plus anciens, les papyrus égyptiens, rapportent l'existence, vers 1350 avant J.C., d'une maladie provoquant l'érection de la verge que les médecins soignaient par des injections dans l'urètre. Des historiens comme Ackerknecht [1] sont convaincus qu'il s'agit de gonococcie. Les livres saints des Israélites, la Thora en particulier, nous donnent plus de précisions et décrivent une blennorragie avec son symptôme capital, l'écoulement purulent. D'après ces textes, avant l'entrée en Terre promise, les Juifs avaient eu des contacts avec d'autres peuplades sémitiques et il se

Correspondance :

Dr Georges ANDROUTSOS - 1 rue Ipeirou, 10433, Athènes, Grèce. Email lyon 48@otenet.gr 
répandit parmi eux une affection provoquant un écoulement génital, dont la contagiosité ne fait pas de doute. Cela se produisit une première fois vers 1200 avant J.C. ; les autorités prirent une mesure draconienne, qu'ils croyaient radicale : l'éradication de tous ceux qui en étaient atteints. Une autre fois, la maladie survint lors de la captivité de Babylone (72 av. J.C.) [11].

La gonorrhée est ainsi décrite au 15e chapitre du Lévitique rédigé au $5^{\mathrm{e}}$ siècle avant notre ère « ...Tout homme qui a une gonorrhée est par la même impur. C'est à cause de sa gonorrhée qu'il est impur : que sa chair laisse couler son flux, ou qu'elle le retienne, il est impur...» [39].

Une allitération biblique a fait confondre gonorrhée et gomorrhée par allusion aux villes de Sodome et Gomorrhe, et aux dépravations sexuelles auxquelles leur nom est lié.

Pendant l'Antiquité, l'insertion dans l'urètre d'un instrument ressemblant à un cathéter aurait été préconisée pour rétablir le passage de l'urine « bloquée » par l'obstruction due à la maladie. On a également découvert des instruments métalliques datant de l'époque probablement destinés au même usage. On en trouve mention dans plusieurs documents anciens [51].

Les citations sont nombreuses dans l'Antiquité grécolatine [56] aussi bien que dans les textes des anciens Hindous et Chinois. Hippocrate (460-377) pensait après avoir ouvert des urètres infectés qu'elle était due à des carnosités, ce terme qui ne veut rien dire et que l'on retrouve obsédant jusqu'à la fin du $19^{\mathrm{e}}$ siècle. II signifiait aussi bien granulomes inflammatoires que polypes, tumeurs, ou rétrécissements. Hippocrate s'exprimait ainsi : " ceux qui ont des tubercules ou des carnosités dans l'urètre en guériront par la suppuration et l'écoulement du pus " [31]. Son optimisme était désarmant car on savait depuis des siècles que la maladie pouvait donner entre autres des redoutables rétrécissements de l'urètre. Sénèque ( 55 av. J.C.- 39 apr. J.C.), au cours d'une crise de rétention due à un de ces rétrécissements, s'ouvrit les veines dans son bain, n'en supportant plus de souffrir [36].

Aulus Cornelius Celsus (42 av. J.C. - 38 apr. J.C.) décrit certaines complications, en particulier les funiculites et les orchi-épididymites [15].

Galien (129-201) a été le premier à employer le mot gonorrhée pour désigner cette affection qu'il considérait comme une « une sécrétion involontaire de semence corrompue " [25]...On retrouve cette erreur jusqu'au $19 \mathrm{e}$ siècle.
Au Moyen Age, la maladie était bien connue des médecins islamiques Rhazès (850-930) [49] et Avicenne (980-1037). La médecine perse, avec Ali Abbas (930995), distingue l'écoulement de sperme et l'écoulement urétral purulent, sans s'attacher à sa contagiosité. Plus tard, Oseibiah (1203-1273) attribue l'écoulement purulent à un rapport sexuel impur avec un animal. II cite un cas de " corde de l'urètre " provoqué par des caroncules intra-urétraux. Le médecin l'aurait traitée par un coup de poing sur la verge posée sur une pierre ! Parmi les occidentaux, Guillaume de Salicet (1210-1277) dans sa Cyrurgia décrit parfaitement la gonorrhée [26].

Le nom de gonorrhée donné à la maladie n'a en effet pas le même sens que celui de gonorrhoea des auteurs grecs ou latins qui signifie " écoulement de la semence», c'est-à-dire spermatorrhée ou leucorrhée et non émission purulente [54].

Le nom blennorragie fut donné, vers 1800 , par François Xavier Swediaur (1748-1812) pour désigner la maladie appelée jusque-là gonorrhée ou dans le langage populaire écoulement, échauffement, chaude-pisse, goutte militaire, etc.

Une petite minorité d'écrivains affirme que la blennorragie n'était pas connue dans l'Antiquité $[47,48,45]$.

\section{UNICITE OU DUALITE ?}

La syphilis fut confondue avec la gonorrhée qui était pourtant connue depuis les temps les plus reculés. Cette étrange confusion entre deux maladies pourtant si différentes provient sans doute de leur coexistence fréquente durant les six derniers siècles où la nosologie fit des progrès très lentement [36]. La confusion la plus totale entre la gonorrhée et la syphilis a persisté pendant plusieurs siècles, donnant lieu à de vives polémiques entre partisans de la dualité de deux grandes maladies vénériennes et ceux qui " prêchaient " leur unicité.

Lors de l'explosion de la grande pandémie de syphilis en Europe, elle ne sera pas confondue avec le nouveau fléau par les médecins contemporains, mais dès le milieu du $16^{\mathrm{e}}$ siècle, la blennorragie deviendra un des symptômes de la vérole, ce qui peut s'expliquer par le fait que les deux affections coexistaient souvent. En 1536, Antonio Musa Brassavola (1500-1555) dans son traité Examen simplicium medicamentorum [10] écrit que la gonorrhée est la première phase de la syphilis, mais reconnaît qu'il existe une forme non-syphilitique de l'inflammation urétrale et cite 234 syndromes du morbus gallicus, dont la gonorrhoea, une species morbi gallici. Il soutient néanmoins qu'elle a son propre contagium. Théophraste Paracelse (1493-1541) confond lui aussi blennorragie et syphilis dans une même maladie 
französische gomorrhea. Son erreur se perpétuera pendant trois siècles et demi.

Gabriel Falloppio (1523-1562), appelle gonorrhoea une maladie nouvelle, apparue depuis quelques années, et distingue une gonorrhoea gallica et une autre non gallica. Leonardo Botallo (1530-1580), à Paris, fait la même différence. II sépare aussi verrucae gallicae et verrucae non gallicae que la plupart des auteurs, comme Girolamo Fracastoro (1478-1553) [22], comptent parmi les symptômes de la syphilis sive morbus gallicus.

Thierry de Héry (Theodoricus) (1505-1585) [55], grand spécialiste en maladies vénériennes, tombe avec Ambroise Paré (1509-1590) [5] dans la même confusion, bien qu'il reconnaisse qu'il existe une forme aiguë de chaude-pisse qui constitue une entité à part. Ils admettent que la maladie surgit le plus souvent après des copulations impures, ils acceptent aussi qu'elle peut se développer après des excès alimentaires, une abstinence sexuelle trop longue car alors le sperme en rétention peut entrer en putréfaction, ou au contraire après une activité sexuelle excessive. Beaucoup d'auteurs, dont André Vésale (1514-1564) [4] et Thomas Bartholin (1616-1680) [36], constatent la participation prostatique fréquente des affections gonorrhéïques. Mais la confusion reste totale entre gonorrhée, syphilis, urétrites non gonococciques, prostatites banales et spermatorrhée. La notion d'unicité des maladies vénériennes prévaut pendant plus de deux siècles.

Le $17 \mathrm{e}$ siècle sera celui d'une confusion totale entre blennorragie, syphilis et spermatorrhée, mais au milieu du 18e siècle Giovanni Battista Morgagni (1682-1771) [41], commencera à débrouiller le problème. II montrera que l'écoulement urétral ne dépend pas d'un ulcère du canal et que l'écoulement est fourni, non pas par les glandes séminales, mais par la muqueuse de l'urètre. John Hunter (1728-1793) [2] soutiendra d'abord cette opinion, mais voulant s'inoculer la blennorragie avec du pus urétral il s'inoculera, en 1767, la syphilis (le malade ayant les deux affections), ce qui le fera conclure à l'identité de ces maladies [33].

En 1736, Jean Astruc (1684-1766) dans son célèbre traité $D e$ Morbis Venereis [6] maintient toujours que la gonorrhée est une complication de la syphilis.

Vers le $18^{\mathrm{e}}$ siècle, certains médecins se posent la même question et en arrivent à distinguer une gonorrhée galénique, maladie non vénérienne, et une gonorrhée vénérienne, symptôme d'une affection constitutionnelle : En 1766, Francis Balfour [7] écrit : « Presque tous les écoulements urétraux sont vénériens et contagieux, mais la gonorrhée et la syphilis sont deux maladies entièrement différentes pour lesquelles il existe deux poisons spécifiques».
Le mérite d'avoir établi clairement que la syphilis et la gonorrhée sont deux maladies distinctes, revient à Benjamin Bell (1749-1806) [9], qui en 1793 introduit dans l'urètre d'un patient un stylet chargé de la matière d'un chancre et chez un autre met en place entre le gland et le prépuce de la charpie imbibée de la matière de la gonorrhée. II démontre ainsi la différence entre le virus de la gonorrhée et celui de la syphilis. En l'espace de 5 ans, Bell réalise une soixantaine d'inoculations et il remarque qu'en inoculant le pus blennorragique à un sujet sain, on n'observe pas de manifestations cliniques propres à la syphilis. II invente le terme de blennorragie formé des mots grecs blennos = mucus et le radical rheo $=$ couler .

La preuve définitive de la dualité est apportée par Joseph François Hernandez (1769-1835) en 1812 et surtout en 1838 par Philippe Ricord (1800-1889) (Figure 1).

Le mémoire de Hernandez intitulé Essai analytique sur la non-identité des virus gonorrhéïques et syphilitiques

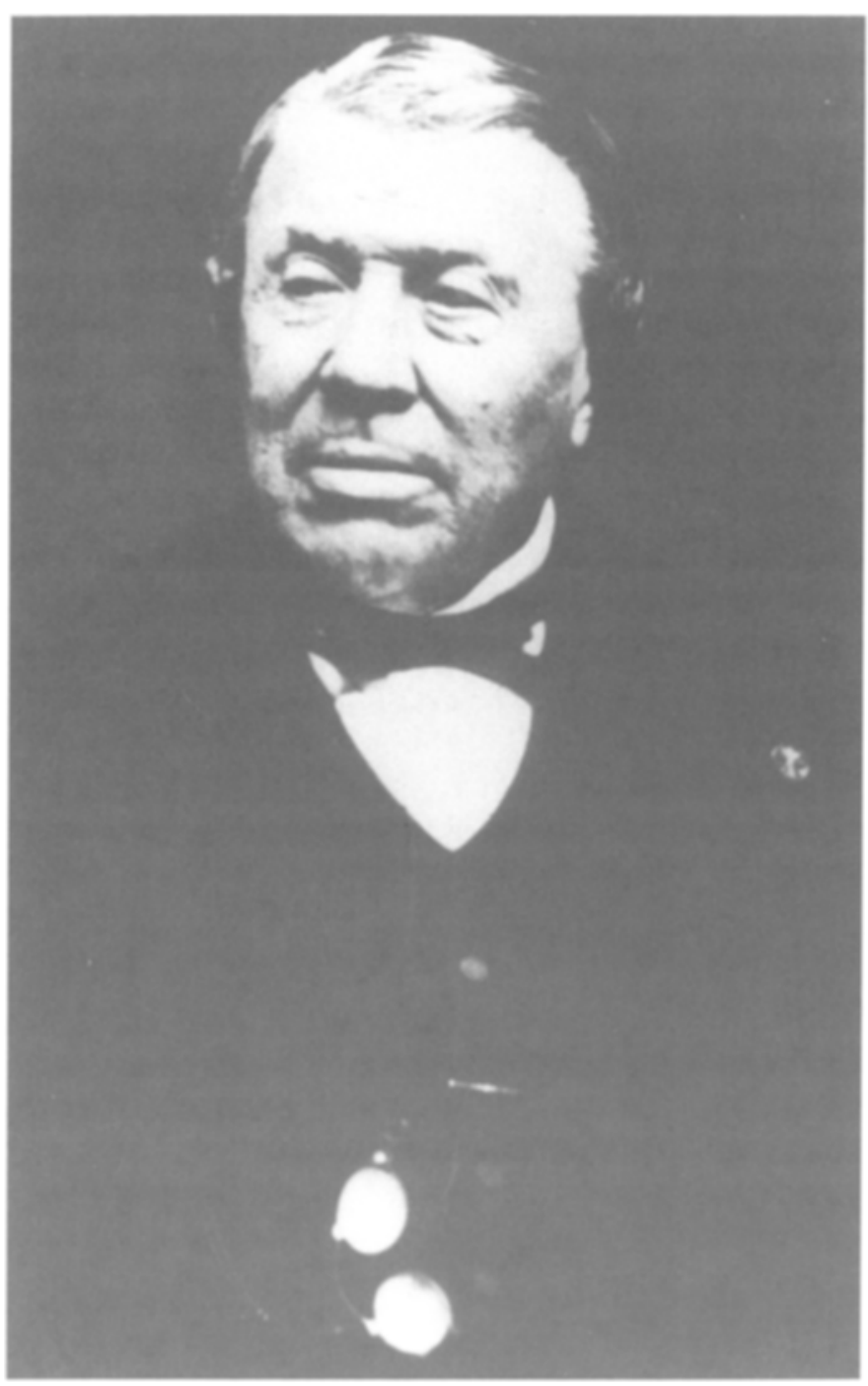

Figure 1 : Philippe Ricord (1800-1889). 
[29], fut couronné le 3 juillet 1812 au concours de Besançon. Trois forçats du bagne de Toulon en avaient fait les frais : " Je les gardai trois mois, dit-il, et c'est pendant ce temps que de nombreuses expériences ont eu lieu... Trois hommes, bien sains et à la force de l'âge, furent inoculés à plusieurs reprises, au gland et au prépuce. L'inoculation se faisait en plaçant sur l'incision, à la lancette, plusieurs fils bien trempés dans la matière gonorrhéique. On eut toujours des ulcères légers sans apparences chancreuses, qui guérirent facilement par les pansements les plus simples. "Plusieurs expériences effectuées chez 17 autres forçats, relatés par Hernandez dans des essais connexes, s'affirmèrent aussi probants et c'est ainsi qu'il put démontrer la dualité de la maladie vénérienne. Mais en dépit de la sanction méritoire de la Société de Besançon à Hernandez, il fallut attendre encore un tiers de siècle, pour que Ricord jugeât en dernier ressort.

A partir des années 1830 Ricord s'applique à tirer au clair, par la clinique et l'expérimentation, le chapitre confus des maladies vénériennes. Pour ce faire, il a recours à la méthode des confrontations entre partenaires, contaminés et contaminants. II examine les femmes au spéculum, dont il met au point un nouveau type. Ricord [50] pratiqua plus de 650 inoculations et établit sans appel possible, dans son Traité de l'inoculation (1838) que la blennorragie doit être séparée de la syphilis : « Un contagium vénérien produit toujours des chancres quand on l'a prélevé sur un premier chancre. L'écoulement urétral inoculé sous la peau ne produit aucune lésion. II faut donc séparer la blennorragie du mal vénérien mais, néanmoins, elle est parfois inoculable, comme toute inflammation».

Les publications favorables ou opposées à l'identité de la syphilis et de la gonorrhée se multiplient. Un des élèves de Ricord, Léon Bassereau (1810-1887) [8], après avoir examiné et suivi minutieusement ses malades, reconnaît en 1852 deux sortes de chancres : un chancre mou et un chancre induré ; Seul ce dernier est suivi des accidents de la syphilis.

En 1843, Pierre Louis Cazenave (1795-1877) [14] écrivait encore : " J'ai vu en un mot, après la blennorragie comme après le chancre, des syphilides graves. J'ai vu la blennorragie être suivie, comme le chancre, d'ulcérations des muqueuses, d'exostoses, de caries des os, et même de cet état tout particulier qu'on appelle la cachexie syphilitique. "

\section{BLENNORRAGIE. UNE MALADIE A PART}

L'agent étiologique de la blennorragie, le germe Neisseria gonorrhoeae, ne fut isolé qu'en 1879 par Albert Neisser (1855-1916) [43] dans le pus et les lésions blennorragiques. Le microbe apparaissait comme de petits granules appariés deux par deux.

En 1876 Emil Noegerrath (1827-1895) [44] décrit la forme latente de la maladie, localisée aux trompes de Fallope chez la femme. L. Leistikow (1882) [37] réussit à cultiver le gonocoque.

En 1885, Ernst von Bumm (1885) [12] obtient des cultures satisfaisantes du gonocoque en utilisant le sérum humain coagulé ; de ses observations, il donne une description détaillée des aspects bactériologiques et histopathologiques. Six ans plus tard, en 1891, Ernst Wertheim, bactériologiste et gynécologue autrichien, parvient lui aussi à isoler en culture pure le gonocoque. Sur les 5 hommes qu'il infecte avec des cultures de gonocoques, tous développent une blennorragie, démontrant ainsi la spécificité pathologique de la bactérie et l'identité nosologique de la maladie. II met ainsi définitivement fin à une longue histoire ayant fait couler beaucoup d'encre.

En 1885, Albert Fraenkel (1848-1916) [23] prouva le rôle du gonocoque dans la vulvo-vaginite infantile et en 1888, Emil Finger [21] publia une monographie sur la blennorragie et ses complications. En 1906, R. Müller et $M$. Oppenheim [42] mirent au point un test diagnostique par fixation de complément.

\section{LOCALISATIONS EXTRAGÉNITALES}

Parfois les gonocoques pénétrant dans les articulations y provoquent une arthrite. En 1893, c'est E. Höck [32] qui montra, le premier, cette localisation, l'existence simultanée de blennorragie urogénitale et d'arthrite ayant été déjà remarquée dès le $16 \mathrm{e}$ siècle et confirmée au siècle dernier par Alfred Fournier (1832-1914) [3].

L'ophtalmia neonatorum était jadis fréquemment observée chez des nouveau-nés dont la mère était atteinte de blennorragie urogénitale et entraînait le plus souvent la cécité. Cette ophtalmie fut déjà décrite en 1722 par Saint-Yves [52], qui croyait que l'infection cheminait par voie sanguine de l'urètre à l'œil. Plus tard il fut démontré que c'est par les voies génitales maternelles que le nouveau-né était infecté [57].

Les gonocoques pénètrent parfois dans l'endocarde où ils peuvent provoquer une endocardite et une septicémie. Ces formes très graves d'infection à $N$. gonorrhoeae furent décrites par W. C. Thayer et G. A. Blumer (1896) [53].

\section{UNE MALADIE SEXUELLEMENT TRANSMISSIBLE}

Au 13e siècle, Guillaume de Saliset (1210-1277) est le premier à décrire dans sa Cyrurgia la blennorragie 
comme une maladie sexuellement transmissible. A la fin du Moyen Âge, il devient pratiquement évident pour les médecins que la blennorragie est une maladie vénérienne présentant un caractère contagieux.

Contrairement à ce que l'on pourrait croire, le préservatif est loin d'être une invention des temps modernes car il était déjà utilisé avant notre ère, sous une forme différente.

Certaines gravures préhistoriques, de même que des peintures égyptiennes datant de 6000 ans, montrent des scènes où un homme nu a le pénis recouvert d'une " protection ". Dans la Rome antique, des cæcums semblent avoir été utilisés comme moyens contraceptifs, mais aussi pour se prémunir des maladies vénériennes. Dans la Chine ancienne, on utilisait le yin-kia, préservatif en papier de soie huilé.

Fallope, inventa le premier préservatif masculin. C'est au $18^{\mathrm{e}}$ siècle que les préservatifs modernes destinés à se préserver de la blennorragie et autres maladies vénériennes, seront fabriqués à partir d'intestins de moutons, de vessies de porcs.

À partir de 1875 , le caoutchouc sera utilisé par les Anglais (d'où le nom de capote anglaise) pour la fabrication du préservatif. C'est au $20^{\mathrm{e}}$ siècle qu'apparaît le préservatif en latex [27].

Paré, en 1560, évoque, tout comme Brassavola en 1553 , un contagium spécifique, origine de la chaudepisse. Mais cette notion n'est pas admise par tous. Quelques-uns croyaient avoir remarqué une generatio spontanea à la suite de rapports trop fréquents ou contre nature. Même la position dans laquelle a lieu la copulation peut avoir son importance. Giorgio Baglivi (1669-1707), en 1700, affirme avoir constaté que la blennorragie est survenue fréquemment chez l'homme après un contact avec une femme saine. Cette croyance persistera encore longtemps et par exemple Ricord écrit en 1860 : « Fréquemment les femmes donnent la blennorragie sans l'avoir » ; et Fournier pense, en 1866 , que " l'homme se donne la chaude-pisse plus souvent qu'il ne la reçoit "».

Alors que certains médecins sont convaincus de la contagiosité de la maladie, d'autres la minimisent d'une façon surprenante. Ainsi Ricardus Anglicus conseille, en 1250 : " en cas de douleurs et d'une forte inflammation de la verge, d'avoir un rapport fort prolongé ; pendant ce temps le vagin ramollit l'inflammation, vide la sécrétion et la purifie ". Trois cents ans plus tard, Hercules Saxonia (1555-1607) rapporte l'expérience de vénitiens avertis. Pour se débarrasser d'une blennorragie, ils avaient dès qu'elle se déclarait, des relations sexuelles avec une Négresse. Selon ses observations, il aurait souvent constaté «la guérison d'une urétrite après un coït avec une vierge ". De même, Samuel Hafenreffer (1587-1660) enseigne que la blennorragie peut guérir par un rapport avec une fille innocente. C'est une opinion encore courante dans le peuple et il est fréquent que des malades hypersexuels perpètrent un viol dans l'intention de se débarrasser d'une infection vénérienne.

Michael Scotus (1214-1291) donne l'avertissement suivant: « Si une femme présente un écoulement, elle infecte la verge de son partenaire. Dans ce cas, la conception entraîne un fruit avarié. L'homme doit donc s'abstenir de tout rapport et la femme doit lui résister avec sagacité ". Les médecins d'Occident, eux aussi, se rendent compte de l'origine de l'écoulement. Guy de Chauliac (1300-1368) [16] cite toutes les complications que peuvent entraîner les relations avec une femme malade : excoriations, brûlures, ulcères virulents, putrides, corrosifs, rongeants, néo-formations carnées, ficus et condylomata. Il envisage ainsi l'étiologie d'une bonne partie de la vénéréologie moderne : la blennorragie, les chancres, les papillomes vénériens.

Le Regimen Salernitanum (1400) résume à sa façon, dans une poésie, les mesures prophylactiques. II conseille les rapports entre personnes mariées. Celui qui outrepasse ce sage avertissement s'expose à toutes sortes de maux. Pour les éviter, il recommande une bonne toilette des organes génitaux, tant masculins que féminins, avant et après le rapport. La miction post coitum est une bonne manière pour préserver l'urètre.

Alessandro Benedetti (1460-1525) insiste sur les nombreux cas de blennorragie (1497).

\section{TRAITEMENT}

Dans l'Antiquité et au Moyen Age, les traitements relèvent de la fantaisie la plus pure. En dehors de mesures anti-phlogistiques externes, certains préconisent la saignée, le vomitif et le sirop de Rosat, d'autres l'injection d'huile de scorpion ou de fiente de colombe détrempée de lessive. Au $9^{e}$ siècle, Rhazès dans une approche un peu plus rationnelle préconise des instillations d'eau miélées, que l'on remplace plus tard par du lait de vache ou de chèvre, ou mieux encore par du lait de femme. A juste titre, la prophylaxie préoccupe les grandes écoles comme celle de Salerne qui recommande après chaque rapport suspect de se laver avec de l'eau vinaigrée, voire avec sa propre urine si l'on n'a rien d'autre [36].

Comment alors s'en protéger ? L'Église préconise purement et simplement la chasteté, mais très peu de moyens sûrs et offrant une certaine sécurité sont disponibles : quelques "recettes", des 
recommandations et des précautions.

Jean Fernel (1497-1558) [20] donne une excellente description de la blennorragie et de ses complications. Comme traitement, il cite des lavages à l'aide d'un siphon ou d'une sonde, et dans certains cas il conseille l'introduction de bougies de cire dans l'urètre. Parmi les complications, il signale la formation de carnosités (caronculae, caroncules) dans le col de la vessie. Leur traitement consiste en l'introduction d'une sonde qui devait rester jusqu'à vingt heures en place, fixée extérieurement par une corde passée autour des lombes.

Parmi les rares recettes relevées dans la littérature, figure celle de Guillaume de Saliset. II indique que « celui qui veut sauver son membre de toute corruption doit le laver avec de l'eau vinaigrée quand il vient de voir une femme qu'il soupçonne infectée d'impudicité ». Un autre médecin recommande l'injection d'un liquide " composé d'eau de chaux, de miel rosat et de mercure doux ». Au 19e siècle, les huiles balsamiques seront utilisées pour combattre l'infection gonococcique et, à partir de 1892, le permanganate de potassium.

Jusqu'à la fin du 19 e siècle on ne connaît guère les médications internes, où le santal, sans doute le premier antiseptique urinaire, fait son apparition. II existait bien une médication abortive interne, la fameuse potion de Chopart, préparation complexe et ancienne dans laquelle prédominait le baume de Copahu. C'était en fait une drogue abominable qui exposait les malades à des accidents cholériformes et qui n'avait aucune efficacité [46].

Les thérapeutiques consistaient en cataplasmes et traitements anti-phlogistiques externes, et en instillations de toutes espèces, aussi variées qu'inefficaces ou dangereuses par leur causticité. La terrible « chaudepisse cordée " n'est pas rare. Elle est horriblement douloureuse car l'urètre et le corps spongieux enflammés perdent leur élasticité et se tendent comme une corde d'arc durant l'érection qui cause des fissures de la muqueuse. Les complications de prostatites et d'orchites sont fréquentes, elles passent le plus souvent à la suppuration. Les arthrites et les endocardites sont habituellement mortelles [36].

De Christmas en 1897, puis von August Paul Wassermann (1866-1925) en 1906, et beaucoup d'autres après, s'essayèrent à l'obtention d'un sérum antigonococcique. C'est à sir Almroth Edward Wright (1861-1947), en l'année 1903, qu'il faut rapporter les premiers efforts de vaccinothérapie de la chaude-pisse. Il été suivi, depuis, par d'innombrables émules. Mais c'est à Rubinstein (1921) et Gauran (1923), qu'en revient la technique la plus parfaite et la plus large divulgation. Le traitement local « antiparasitaire ", que pratiquait Paul Charles Diday (1812-1894) en 1884, fut augmenté par ses soins d'une cure abortive, en 1891. Neisser, dès sa découverte du germe, et surtout entre 1895 et 1907, usait du lavage urétral (Figure 2) comme du meilleur procédé d'antisepsie. En fait, c'est Janet qui mit au point en 1892 le traitement local de la gonococcie par les lavages de l'urètre effectués sous pression, à l'aide d'une seringue à embout en mousse (Figure 3 ) qui porte son nom, avec une solution diluée de permanganate de potasse. Répétés trois fois par jour, ils permettaient la guérison en quatre semaines, sans cependant mettre à l'abri des complications et, en particulier, de l'orchiépididymite et des séquelles tardives que constituait le rétrécissement urétral, traité par des dilatations à l'aide des béniqués [46].

Le procédé de Janet fut aussi utilisé à titre prophylactique, avant l'apparition d'un écoulement, chez les sujets qui, après un rapport suspect, craignaient une contamination. Cette méthode permettait sans doute parfois une véritable guérison avant l'apparition de l'écoulement, mais était aussi à l'origine d'urétrites
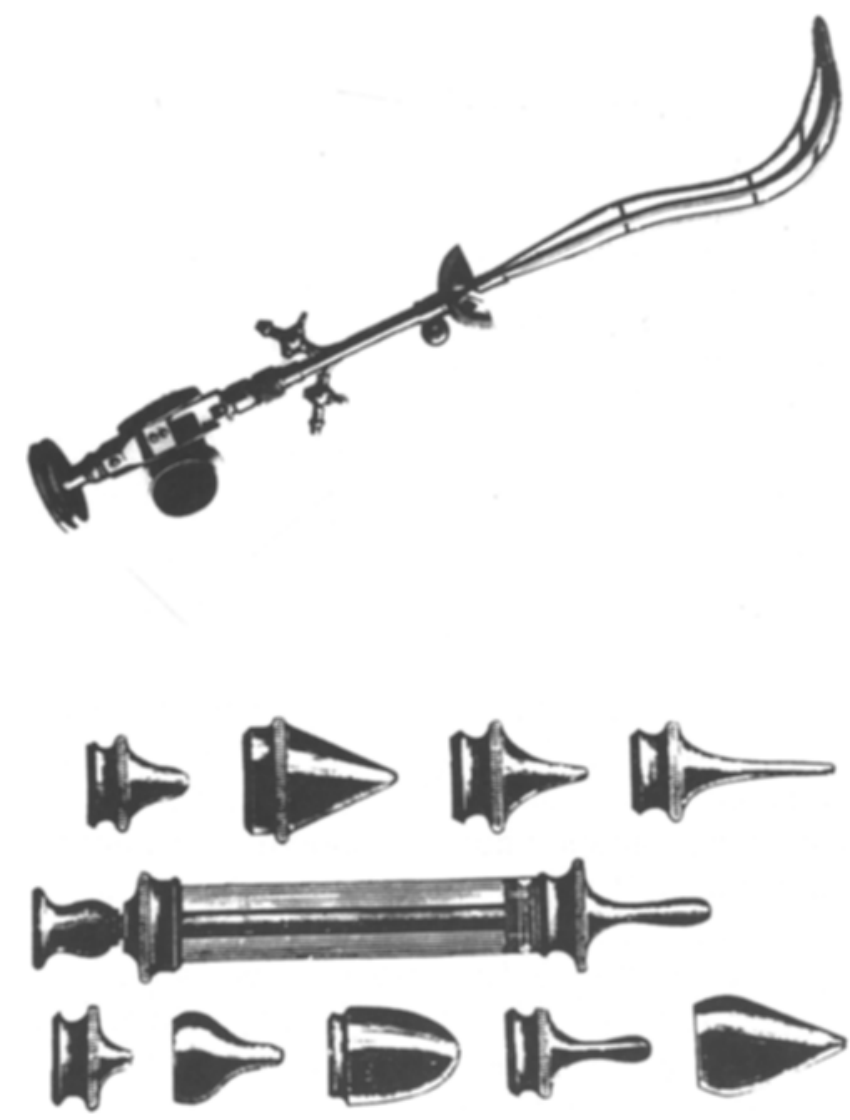

Figure 2 : En haut : seringue individuelle pour instillation urétrale. En dessous : dilatateur laveur. L'appareil utilisé pour le traitement des urétrites postérieures maintient l'urètre largement entrouvert et permet en même temps une irrigation à grand débit. 


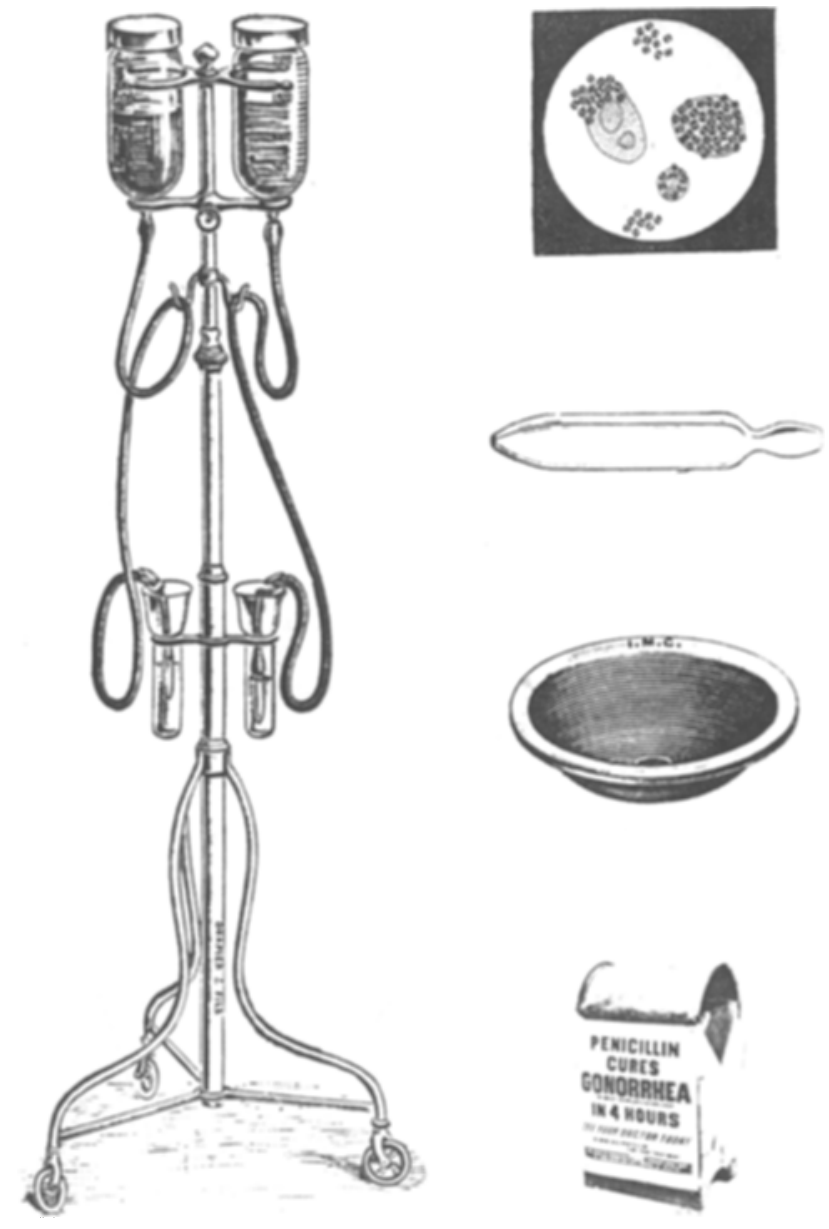

Figure 3 : A gauche : appareil de Janet. A droite de haut en bas : le gonocoque; la canule de Janet; le disque protecteur placé derrière la canule protège l'opérateur ; publicité à New York pour la pénicilline.

chimiques lorsque les instillations étaient poursuivies pendant un temps trop prolongé [36].

La chimiothérapie intraveineuse fit ses débuts, d'abord timides, avec les publications de Duhot, en 1912, de Janet et Lévy-Bing, en 1913 [34].

Durant la guerre de 1914-1918, la recrudescence des maladies vénériennes dans les deux camps prend des proportions inquiétantes. Des mesures prophylactiques et le traitement abortif prennent une importance stratégique. Des instillations de nitrate d'argent sont essayées, mais à trop fortes concentrations. Elles provoquent des réactions intenses, capables d'aggraver la blennorragie naissante. Le protargol, un albuminate d'argent que l'on injecte à la concentration de $4 \%$ est beaucoup mieux toléré. Le rythme est de 2 injections par jour pendant cinq jours ; si au bout de cette période un écoulement apparaît et persiste, le traitement abortif est considéré comme un échec et l'on a recours aux grands lavages.

En 1926 eurent lieu les premières tentatives d'un traitement général de la blennorragie par une chimiothérapie intraveineuse aux sels d'acridine. Paul Ehrlich (1854-1915) a le mérite d'avoir émis l'hypothèse que les colorants qui se fixent sur les parties constituantes des cellules vivantes pouvaient avoir des propriétés antiseptiques. Selon lui, les dérivés du jaune d'acridine étaient relativement bien tolérés par l'organisme. Malgré la disparition des complications génito-urinaires chez les malades ainsi traités, l'inconstante efficacité de cette nouvelle thérapeutique n'entraîna pas l'adhésion unanime des vénéréologues, qui restèrent plus ou moins fidèles à la méthode de Janet. L'acridinothérapie n'était d'ailleurs pas exempte de tout inconvénient ; la sensibilité considérablement accrue aux radiations solaires durant les heures qui suivaient l'injection nécessitait quelques précautions en particulier chez les malades ambulatoires, mais elle avait, en revanche, l'avantage d'amener à une meilleure connaissance de la photosensibilisation, des maladies de lumière et de leur traitement. La trypaflavine fut employée pour le lavage de la plèvre en cas de pleurésie purulente. A partir de cette dernière, Jausion utilisa la gonacrime ; injectée par voie intraveineuse, elle se montra efficace, malgré les incidents de photosensibilisation que nous venons d'évoquer et les dangers de sclérose veineuse [13].

En 1936, la découverte des sulfamides transforme complètement la thérapeutique et le pronostic, et les grands lavages sont progressivement abandonnés. L'action des sulfamides sur le gonocoque est démontrée par Constant Levaditi (1874-1953) et A. Vaisman (1937) [38] chez la souris, et par J.E. Dees et J.A. Colston (1937) [18] chez l'homme.

En 1943, la pénicilline [30] prend le relais d'une sulfamidothérapie devenue défaillante par sulfamidorésistance, et ultérieurement les tétracyclines, les céphalosporines, etc., pallieront par la suite à la penicillinorésistance.

La prévention de l'ophtalmie gonococcique par l'instillation de nitrate d'argent dans l'œil des nouveaunés, déjà préconisée en 1835 par E.F. Julliard [35], fut systématiquement appliquée en 1884 par Karl Siegmund Franz Crédé (1819-1892) [17] et amena une diminution considérable du risque de cécité [24].

L'action de la chaleur sur les gonocoques motiva l'utilisation de l'hyperthermie dans le traitement de l'arthrite gonococcique [58, 19, 40].

Aujourd'hui, la prévention de la blennorragie passe par l'utilisation du préservatif, et sa thérapeutique par le recours aux antibiotiques. En 1997, on enregistrait 62 millions de nouveaux cas de blennorragie, dont une très faible proportion était mortelle. 


\section{REFERENCES}

1 ACKERKNECHT E. : Geschichte und Geographie der wichtingsten Krankheiten. Stuttgart, Enke, 1963 : 107.

2. ANDROUTSOS G. : L'andrologie dans les CEuvres complètes de John Hunter (1728-1793), fondateur de la chirurgie scientifique. Andrologie, 2003, $13: 465-473$.

3. ANDROUTSOS G., VLADIMIROS L. : Le fondateur de la syphiligraphie Alfred Fournier (1832-1914) et sa lutte contre la syphilis. Andrologie, 2007, $17: 80-87$.

4. ANDROUTSOS G. : L'urologie dans les planches anatomiques d'André Vésale (1514-1564). Prog. Urol., 2005, $15: 544-550$.

5. ANDROUTSOS G. : Ambroise Paré (1509-1590) andrologue. Andrologie, 2001, $11: 258-262$.

6. ASTRUC J. : De Morbis Venereis. Montpellier, 1736.

7. BALFOUR F. : Dissertatio de gonorrhoea virulenta. Edimbourg, 1767.

8. BASSEREAU L. : Affections de la peau symptomatiques de la syphilis. Paris, Baillière, $1852: 36$.

9. BELL B. : Treatise on gonorrhoea virulenta and lues venereal. 2 vol., Edimbourg, 1793.

10. BRASSAVOLA A.M. : Examen simplicium medicamentorum. Rome, 1536.

11. BRODNY M. : The history of gonorrhea among the Greeks and Romans. Trans. Amer. Neisser. Med. Soc., 1937, 3 ; 92106.

12. BUMM E. von : Der Mikro-organismus der gonorrhoischen. Schleimhaut-Erkrankungen, Gonococcus- Neisser. Disch. Med. Wschr., 1985, $11:$ 508-509.

13. BURGUN R., LAUGIER P. : Histoire des maladies vénériennes. In : Histoire de la médecine, de la pharmacie, de l'art dentaire, et de l'art vétérinaire. Collection dirigée par J. Poulet, J.C. Sournia et M. Martiny en 8 vol. Paris, Albin Michel-Laffont-Tchou, 1978, t. IV : 283-346.

14. CAZENAVE P.L. : Syphilides. Paris, Doin, 1843.

15. CELSE A.C. : Traité de Médecine. Traduction Véndrènes, Paris, Masson, 1876.

16. CHAULIAC GUY DE : La pratique en chirurgie du maistre Guidon de Chauliac. Lyon, 1478.

17. CREDE K.S.F. : Die Verhütung der Augentzündung der Neugeborenen (ophtalmoblennorhea neonatarum) der haüfigsten und wichtigsten Ursache der Blindheit. Berlin, $1884: 24-26$.

18. DEES J.E, COLSTON J.A.C. : The use of sulphanilamide in gonococcic infections. Preliminary report. J. Amer. Med. Assoc., 1937, 108 : 1855-1858.

19. DOWLING H.F. : Fighting infection, conquests of the twentieth century. Cambridge (Mass) and London, 1978 : 13-15.

20. FERNEL J. : De luis venarae curatione. Paris, 1578.

21. FINGER E.A.F. : Die Blenorrhoea der Sexualorganen und ihre Complicationen. Leipzig et Vienne, $1888: 27$.
22. FRACASTORO G. : Syphilis sive morbus gallicus. Venise, 1530.

23. FRAENKEL E. : Bericht über eine bei Kindern beobachtete Endemie infectiöser Colpitis. Virch. Arch. Path. Anat., 1885, $99: 251-276$.

24. FRIEDENWALD H. : Paths of progress in Ophtalmology. Arch. Opht., 1942, $27:$ 1047-1096.

25. GALIEN : CEuvres médicales choisies. Traduction de Charles Daremberg. Choix, présentation et notes par A. Pichot. 2 vols, Paris, Gallimard, 1994.

26. GRMEK M. : Les maladies à l'aube de la civilisation occidentale. Paris, Payot, $1983: 214-215$.

27. HANSEN W., FRENEY J. : Des bactéries et des hommes. Toulouse, Privat, $2002: 62-65$.

28. HENSCHEN F. : The history and geography of diseases. New York, Delacorte Press, 1966 : 88.

29. HERNANDEZ J.F. : Essai analytique sur la non-identité des virus gonorrhoïque et syphilitique. Toulon, 1812.

30. HERRELL W.E., COOK E.N. : Use of penicillin in sulfonamide resistant gonorrhoeal infections. J. Amer. Med. Assoc., 1943, $22: 289-292$.

31. HIPPOCRATE : CEuvres complètes d'Hippocrate. Commentées et recueillies par E. Littré, 10 vol. Paris, J.B. Baillière et Fils, (1836-1861) Epid., III, 7 ; Morb., II, 51 ; Aph., IV, 82.

32. HÖCK H. : Ein Beitrag zur Arthritis blennorrhoica. Wien. klein. Wschr., 1893, $6:$ 736-738.

33. HUNTER J. : A Treatise on the Venereal Disease. London, Livingstone, 1793.

34. JAUSION H., MEDIONI G. : Histoire du mal vénérien. In : Histoire générale de la médecine, de la pharmacie, de l'art dentaire et de l'art vétérinaire. Sous la direction de LaignelLavastine. 3 vol., Paris, Albin Michel, 1935-1948, t. 2 : 541545.

35. JULLIARD E.F. : De l'emploi de l'excision et de la cautérisation à l'aide du nitrate d'argent fondu dans l'ophtalmie blennorragique. Thèse Méd. $n^{\circ}$ 26, Paris, 1835.

36. KÜSS R., GREGOIR W. : Histoire illustrée de l'urologie. De l'Antiquité à nos jours. Paris, Les éditions Roger Dacosta, 1988: 357-365.

37. LEISTIKOW L. : Über Bacterien bei den venerischen Krankheiten. Charité Ann., 1882, $7:$ :750-752.

38. LEVADITI C., VAISMAN A. : La toxi-infection gonococcique expérimentale et son traitement chimiothérapique. Presse méd., 1937, $45: 371-373$.

39. LEVITIQUE : Ch. 15.

40. MC CAHEY : History of gonorrhoeal arthritis. In : A System of Bacteriology in relation to Medicine. Vol. 2, $1929: 281-82$.

41. MORGAGNI J.B. : De Sedibus et causis morborum per anatomen indagatis. Venise, 1761.

42. MÜLLER R., OPPENHEIM M. : Über den Nachweis von Antikörpen im Serum eines in Arthritis gonorrhoica Erkrankten mittels Komplementablenkung. Wien. Klein. Wschr., 1906, 19: 894-895. 
43. NEISSER A. : Über eine der Gonorrhoe eigentümliche Micrococcusform. Zbl. Med. Wiss., 1879, 17 : 497-500.

44. NOEGERRATH E. : Die latente gonorrhoe in weiblichen Geschlecht. Bonn, 1872 : 46-48.

45 ORIEL J. : Gonorrhoea in the Ancient World. Paleopath. Newsletter, 1973, $4: 14-21$.

46. PARIENTE L. : Petite encyclopédie des maladies sexuellement transmissibles. Histoire et actualité. Paris, Editions Louis Pariente, 1993, 383.

47. PAZZINIA. : De amatorum morbis. Boll. Istit. Stor. Ital. Arte San., 1930, $10: 1-18$ et 131-154.

48. PAZZINI A. : Ancora sulla blenorragia degli antichi. Atti Mem. Stor. Arte San., $1938: 85-90$.

49. RHAZES : Liber nonus ad Almansorem (Continent). Padoue, 1476.

50. RICORD P. : Traité des maladies vénériennes. Paris, Doin, 1838.

51. ROSEBURY T. : Microbes and morals. A study of venereal disease. St Albans, $1975: 24$.

52. SAINT-YVES : Nouveau traité des maladies des yeux. Paris, Gabon, 1722.

53. THAYER W.S., BLUMER G.A. : Ulcerative endocarditis due to the gonococcus; gonorrhoeal septicemia. Johns Hopk. Bull., 1896, $7: 57-63$.

54. THEODORIDES J. : Des miasmes aux virus. Histoire des maladies infectieuses. Paris, Editions Louis Pariente, 1991: 126-129.

55. THIERRY DE HERY : La méthode curatoire de la maladie vénérienne. Paris, 1552.

56. VERTUE H. : Enquiry into venereal disease in Greece and Rome. Guy's Hosp. Rep., 1953, 102 : 277.

57. VETCH J. : A practical treatise on the diseases of the eye. London, Livingstone, $1820: 35-38$.

58. WARREN S.L., WILSON K.M. : Treatment of gonococcal infections by artificial hyperthermia: a preliminary Report. Amer. J. Obst. Gyn., 1932, 24 : 592-598.

59. WERNER A., GOETSCHEL H. : Les épidémies. Un sursis permanent. Tournai (Belgique), Atlande, 1999 :191.

Manuscrit reçu : mars 2007 ; accepté mars 2007.

\section{History of gonorrhoea}

\section{Georges ANDROUTSOS and Lazaros VLADIMIROS}

This paper examines the history of gonorrhoea and its treatment. It is very probably a very ancient disease that was confused with syphilis for centuries.

Key words : gonorrhoea, syphilis, treatment, history 